\title{
Progressive hearing loss and cerebellar ataxia in anti-Ma2-associated autoimmune encephalitis
}

Perda auditiva progressiva e ataxia cerebelar na encefalite autoimune associada ao anti-Ma2

Paulo Victor Sgobbi de Souza ${ }^{1}$, Thiago Bortholin', Wladimir Bocca Vieira de Rezende Pinto ${ }^{1}$, Adrialdo José Santos ${ }^{1}$

A 38-year-old Brazilian man presented with a two-year history of progressive bilateral hearing loss, cerebellar ataxia, emotional lability and hypersexuality. A limbic-brainstem syndrome was suspected and neuroimaging studies performed, disclosing marked brainstem signal changes (Figure). Cerebrospinal fluid analysis disclosed a mild protein increase. During paraneoplastic screening, testicular ultrasonography disclosed a heterogeneous complex mass in the right testicle (Figure) and serum anti-Ma2 antibodies were detected.

Anti-Ma2-associated encephalitis classically emerges in the context of seminomatous and nonseminomatous testicular tumors and presents with a complex spectrum of neurological manifestations ${ }^{1}$, including limbic encephalitis, atypical parkinsonism, cerebellar ataxia, brainstem dysfunction, myelopathy, radiculoplexopathy, REM sleep behavior disorder and narcolepsy ${ }^{1,2}$.
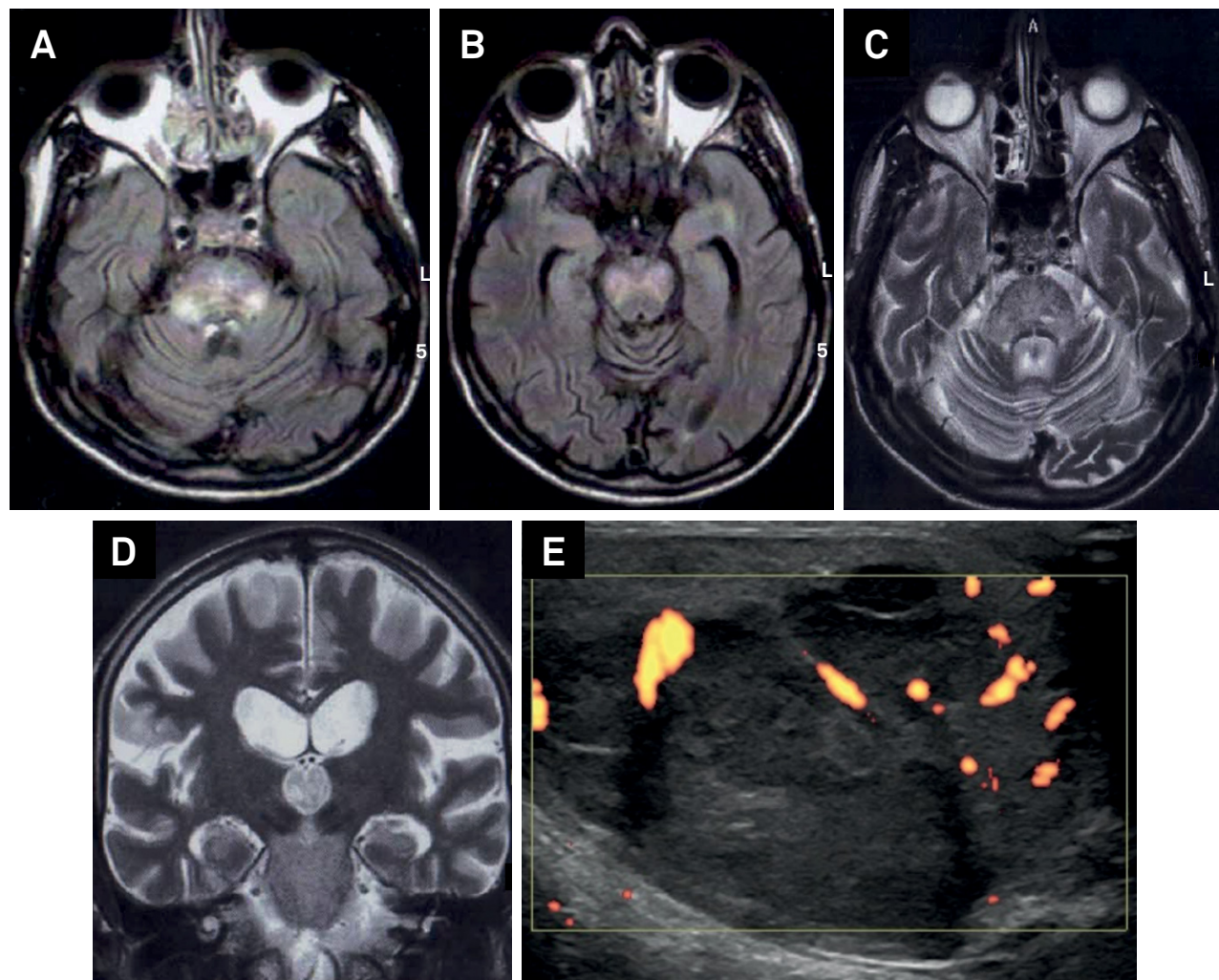

Figure. Brain MR imaging studies disclosing cortical atrophy, bilateral hyperintensity in the mesial temporal lobes and marked hyperintense signal change in the pons and superior cerebellar peduncles in axial FLAIR sequence $(A, B)$ and axial $(C)$ and coronal T2-weighted images (D). (E) Ultrasonography of the testis disclosing a heterogeneous complex mass in the right testicle with the presence of flow inside the lesion in the Doppler study.

${ }^{1}$ Universidade Federal de São Paulo, Departamento de Neurologia e Neurocirurgia, São Paulo SP, Brasil.

Correspondence: Wladimir Bocca Vieira de Rezende Pinto; Departamento de Neurologia e Neurocirurgia da UNIFESP; Rua Pedro de Toledo, 650; 04039-002 São Paulo SP, Brasil; E-mail:wladimirbvrpinto@gmail.com

Conflict of interest: There is no conflict of interest to declare.

Ethical statement: Full consent was obtained from the patient's family for the case report. This study was approved by our Ethics Institution.

Received 11 July 2016; Received in final form 03 September 2016; Accepted 09 September 2016. 


\section{References}

1. Dalmau J, Graus F, Villarejo A, Posner JB, Blumenthal D, Thessen B et al. Clinical analysis of anti-Ma2-associated encephalitis. Brain. 2004;12(8)7:1831-44

doi:10.1093/brain/awh203
2. Mrabet S, Ben Achour N, Kraoua I, Benrhouma H, Klaaa H, Rouissi A et al. Anti-Ma2-encephalitis in a 2 year-old child: a newly diagnosed case and literature review. Eur J Paediatr Neurol. 2015;19(6):737-42. doi:10.1016/j.ejpn.2015.06.005 